\title{
Resultados en el tratamiento del tumor de cuerpo carotídeo, experiencia de 20 años
}

\author{
Héctor Gurrola-Machuca ${ }^{1 *}$, Arturo P. Miranda-Aguirre ${ }^{1}$ y Héctor Gurrola-Luna ${ }^{2,3 *}$ \\ ${ }^{1}$ Servicio de Cirugía Oncológica, Sección de Tumores de cabeza y cuello, Centro Médico Nacional 20 de Noviembre; ${ }^{2}$ Programa de Servicio Social, \\ Universidad Nacional Autónoma de México; ${ }^{3}$ Departamento de Cardiología Nuclear, Instituto Nacional de Cardiología "Ignacio Chávez". Ciudad de \\ México, México
}

\section{Resumen}

Objetivo: Presentar los resultados en el tratamiento de una serie de casos de tumores del cuerpo carotídeo (TCC). Material y métodos: Estudio retrospectivo en el que se analizaron los casos de TCC de pacientes tratados durante 20 años. Se analizó la frecuencia de presentación de acuerdo con la clasificación de Shamblin, el tipo de tratamiento utilizado, las complicaciones y los resultados obtenidos. Resultados: Se incluyeron 55 pacientes con 62 TCC. De acuerdo con la categoría de Shamblin, encontramos 4 casos (6.5\%) tipo I, 44 casos (71\%) tipo II y 14 casos (22.6\%) tipo III. Se realizó cirugía en el $64.5 \%$ de los tumores. La mediana en el sangrado transoperatorio fue de $350 \mathrm{ml}$, el tiempo operatorio fue $120 \mathrm{~min}$. Se presentaron 3 complicaciones posoperatorias (7.5\%), que incluyeron un síndrome de Horner, una lesión del nervio laríngeo superior y un evento vascular cerebral. En el resto de las pacientes el tratamiento fue con radioterapia o vigilancia. Conclusiones: La cirugía es la mejor opción de tratamiento de los TCC, especialmente en los Shamblin I y II. La embolización preoperatoria no fue necesaria para la resección de estos tumores. La radioterapia parece ser el mejor tratamiento en tumores Shamblin III.

Palabras clave: Tumor del cuerpo carotídeo. Paraganglioma. Quimiodectoma. Embolización terapéutica.

\section{Outcomes in the treatment of carotid body tumor, 20 years of experience}

\section{Abstract}

Objective: To present the results of the treatment of a series of cases of Carotid Body Tumors (CBT). Material and methods: Retrospective study in which the cases of CBT of patients treated for 20 years were analyzed. The frequency of presentation was analyzed according to the Shamblin classification, the type of treatment used, complications, as well as the results obtained. Results: 55 patients with 62 CBT were included. According to the Shamblin category, we found 4 cases (6.5\%) type I, 44 cases (71\%) type II and 14 cases (22.6\%) type III. Surgery was performed in $64.5 \%$ of tumors. The median intraoperative bleeding was $350 \mathrm{ml}$, while operative time was $120 \mathrm{~min}$. There were 3 postoperative complications (7.5\%), which included one Horner syndrome, one superior laryngeal nerve lesion and one stroke. In the rest of the patients, treatment was with External Beam Radiation Therapy (EBRT) or surveillance. Conclusions: Surgery is the best treatment option for CBT, especially in Shamblin I and II. Preoperative embolization was not essential for resection of these tumors. EBRT appears to be the best treatment for Shamblin III tumors.

Key words: Carotid body tumors. Paragangliomas. Chemodectomas. Therapeutic embolization.

\footnotetext{
Correspondencia:

*Héctor Gurrola-Machuca

E-mail: gurrolamah@ prodigy.net.mx (http://creativecommons.org/licenses/by-nc-nd/4.0/).

Fecha de recepción: 18-01-2021

Fecha de aceptación: 20-03-2021

DOI: 10.24875/j.gamo.21000009

Disponible en internet: 16-06-2021

Gac Mex Oncol. 2021;20(3):87-93

www.gamo-smeo.com
} 


\section{Introducción}

Los tumores del cuerpo carotídeo (TCC) son neoplasias poco frecuentes y la mayor parte benignas ${ }^{1}$. Se consideran neoplasias neuroendocrinas de lento crecimiento que surgen de las células de la paraganglia, mismas que se localizan en la bifurcación de la arteria carótida común. Representan el $0.6 \%$ del total de tumores de cabeza y cuello, y hasta el $10 \%$ tiene una presentación bilateral ${ }^{1,2}$. La primera clasificación descrita es la que propuso Shamblin en 1971, la cual hasta la fecha sigue siendo la más utilizada ${ }^{3}$. Se trata de una clasificación quirúrgica-patológica que considera el involucramiento de la adventicia de los vasos carotídeos. Para el diagnóstico de esta patología la exploración física es parte fundamental y los estudios de imagen, como el ultrasonido y la tomografía, sirven para confirmar la sospecha clínica. El tratamiento mas recomendado es la resección quirúrgica. Las complicaciones posquirúrgicas más frecuentes son la lesión de nervios craneales, siendo el nervio hipogloso (9.6\%), el vago (8.1\%), el síndrome de Horner $(2.9 \%)$ y el nervio facial (2.8\%) los más afectados. Otras complicaciones importantes son eventos cerebrales isquémicos $(3.5 \%)$, hematomas $(5.2 \%)$ y muerte $(2.2 \%)^{4}$. Se ha descrito que la probabilidad de resección y la tasa de complicaciones se asocia a la clasificación de Shamblin, de forma que para los tumores tipo I y II es más probable lograr la resección con pocas complicaciones, en comparación con los tumores tipo III, para los cuales incluso llega a ser necesario la necesidad de colocación de injertos vasculares. Se ha evaluado también la utilidad de la radioterapia como tratamiento primario, combinado o de rescate en $\mathrm{TCC}^{5}$. Por su parte, la vigilancia representa otra opción para algunos casos $^{6}$.

Hasta la fecha, existe debate de la utilidad de la embolización antes de la resección quirúrgica con el objetivo de disminuir el sangrado transoperatorio e incluso las complicaciones posteriores ${ }^{7}$. El objetivo del presente estudio fue describir los resultados en el tratamiento de una serie de casos del TCC.

\section{Material y métodos}

Se revisaron los expedientes de pacientes que fueron enviados a un servicio de tumores de cabeza y cuello en un hospital de tercer nivel de atención, con diagnóstico de TCC y que cumplieron con los siguientes criterios: mayores de 18 años, tratados durante el periodo de tiempo comprendido entre 1991 al 2019, seguimiento por al menos seis meses, contar con expediente clínico completo y estudios de imagen que confirmaron la presencia de TCC. Se analizaron los siguientes datos clínicos: edad, sexo, tamaño tumoral, el cual fue evaluado con la tomografía preoperatoria, el tipo de tratamiento otorgado, la categoría de acuerdo con la clasificación de Shamblin, el tiempo quirúrgico en minutos, el sangrado transoperatorio y las complicaciones del tratamiento. La técnica quirúrgica utilizada para la escisión del TCC fue similar a la descrita previamente en la literatura ${ }^{8}$ y se utilizó para todos los pacientes, la cual brevemente consiste en incisión cervical, disección de colgajos subplatismal, disección y retracción del esternocleidomastoideo, localización y referencia de la carótida común; posteriormente, disección y referencia de la carótida interna y la externa, disección de la adventicia, iniciando por la carótida interna y continuando con la de la carótida externa; se identifican y respetan los nervios hipogloso y neumogástrico, se identifica, pinza y liga el vaso nutricio principal localizado en la bifurcación, se realiza hemostasia, colocación de drenaje cerrado y cierre por planos. En todos los pacientes llevados a cirugía, el reporte final de patología confirmó el diagnóstico. Se describen los resultados de este estudio transversal, retrospectivo y retrolectivo.

\section{Resultados}

Se incluyeron para su análisis 55 pacientes con 62 TCC, de los cuales 7 casos fueron tumores bilaterales (12.7\%). Las mujeres representaron casi la totalidad de la serie, con 53 casos $(96.4 \%)$. Todos los tumores bilaterales correspondieron al sexo femenino. La media de edad fue de $54 \pm 14.3$ años. La mediana en el tamaño tumoral fue de $4 \mathrm{~cm}$. De acuerdo con la clasificación de Shamblin, 4 casos correspondieron al tipo I (6.5\%), 44 casos al tipo II (71\%) y 14 casos al tipo III (22.6\%). En relación con el tratamiento, se realizó cirugía en 40 tumores (64.5\%), ocho pacientes rechazaron cirugía y en seis el TCC se consideró irresecable por los hallazgos transoperatorios. En ocho pacientes no se realizó cirugía por el alto riesgo quirúrgico debido a comorbilidades, uno de ellos con categoría II de Shamblin y 7 con categoría III. La mediana en el sangrado transoperatorio fue de $350 \mathrm{ml}$ y para el tiempo operatorio fue de $120 \mathrm{~min}$ (Tabla 1). La cirugía fue el tratamiento para todos los casos de Shamblin tipo I, para 35 casos (77.1\%) Shamblin II y un paciente Shamblin III. Se presentaron tres complicaciones posquirúrgicas únicamente $(7.5 \%)$, síndrome de Horner en un paciente, lesión 
Tabla 1. Datos clínicos demográficos de la serie de 55 pacientes con tumores del cuerpo carotídeo (TCC)

\begin{tabular}{|l|c|}
\hline TCC & $\mathrm{n}=62$ \\
\hline Edad, años - Media (DE) & $54( \pm 14.3)$ \\
\hline Sexo, mujer - $\mathrm{n}(\%)$ & $53(96.4 \%)$ \\
\hline Tamaño tumoral, cm - Md (rango) & $4(2.5-8)$ \\
\hline Categoría de Shamblin - $\mathrm{n}$ (\%) \\
I \\
II \\
III \\
\hline Tipo de tratamiento - $\mathrm{n}(\%)$ & $4(6.5)$ \\
Cirugía & $44(71)$ \\
Radioterapia & $14(22.6)$ \\
\hline Vigilancia & \\
\hline Sangrado operatorio, ml - Md (rango) & $40(64.5)$ \\
\hline Tiempo quirúrgico, min - Md (rango) & $10(16.1)$ \\
\hline Estado actual n (\%) & $12(19.4)$ \\
\hline VSAT & $120(40-3,000)$ \\
\hline VCAT & \\
\hline MSAT & $40(64.5)$ \\
\hline
\end{tabular}

DE: desviación estándar; Md: mediana; VSAT: vivo sin evidencia de actividad tumoral; VCAT: vivo con evidencia de actividad tumoral; MSAT: muerto sin evidencia de actividad tumoral.

del nervio laríngeo superior en otro y un evento vascular cerebral (EVC) en otro, mismo que se presentó al cuarto día de la cirugía. En el paciente Shamblin III que fue llevado a cirugía, fue necesario la colocación de injerto vascular sintético. Como era de esperarse, se observó una asociación directamente proporcional entre la categoría del Shamblin y el tiempo transoperatorio y el sangrado (Figs. 1 y 2). La radioterapia fue el tratamiento para 10 pacientes $(16.1 \%)$, tres porque rechazaron cirugía en una categoría Shamblin II, cuatro por considerase irresecables durante la cirugía y otros tres que correspondieron a pacientes con alto riesgo quirúrgico, todos ellos Shamblin III. Doce pacientes (19.4\%) se sometieron únicamente vigilancia, seis con categoría de Shamblin II y seis Shamblin III (Tabla 2, Fig. 3). De los siete pacientes con tumores bilaterales, cuatro se sometieron a cirugía bilateral $(7.3 \%)$, a un paciente se le realizó cirugía de un lado y radioterapia contralateral $(1.8 \%)$, a otro paciente radioterapia y vigilancia contralateral $(1.8 \%)$, y a otro más se le otorgó radioterapia bilateral $(1.8 \%)$. La mediana de seguimiento en esta serie fue de 80 meses (2-226) meses y el desenlace de los pacientes fue: 40 vivos sin evidencia de actividad tumoral (64.5\%), 21 vivos con evidencia de actividad tumoral $(33.9 \%)$ y un muerto sin evidencia de actividad tumoral $(1.6 \%)$.
Tabla 2. Tipo de tratamiento otorgado de acuerdo con la categoría de Shamblin

\begin{tabular}{|l|c|c|c|c|}
\hline \multirow{2}{*}{ Categoría } & \multicolumn{4}{|c|}{ Tipo de tratamiento, n (\%) } \\
\cline { 2 - 5 } & Cirugía & Radioterapia & Vigilancia & Total \\
\hline I & $4(100)$ & $0(0)$ & $0(0)$ & $4(100)$ \\
\hline II & $35(79.5)$ & $3(6.8)$ & $6(13.6)$ & $44(100)$ \\
\hline III & $1(7.1)$ & $7(50)$ & $6(42.9)$ & $14(100)$ \\
\hline
\end{tabular}

\section{Discusión}

En esta serie de casos se presentaron los resultados del tratamiento de 62 TCC, de los cuales la mayoría fueron tratados con cirugía con resultados similares a los reportados recientemente en otros estudios ${ }^{9,10}$. Varios de ellos, incluyendo cinco revisiones sistemáticas, han evaluado la utilidad de la embolización preoperatoria; sin embargo, los resultados de ellos han sido contradictorios en cuanto al verdadero beneficio de este procedimiento ${ }^{7,11-15}$. En la revisión sistemática de Texakadalis, et al. en la que se analizaron 1,326 pacientes, aquellos que se sometieron a embolización preoperatoria tuvieron significativamente menor sangrado (diferencias de medias ponderadas [DMP]: -135.32; intervalo de confianza al 95\% [IC 95\%]: -224.58 a -46.06) y menor tiempo quirúrgico (DMP: -38.71 ; IC 95\%: -65.61 a -11.62). No se encontró diferencia significativa en el riesgo de lesión de nervios craneales, EVC isquémicos y ataques isquémicos transitorios ${ }^{16}$. En otro metaanálisis que incluyó un menor número pacientes también se demostró disminución en el sangrado (DMP: -0.52; IC 95\%: -0.77 a -0.28) y menor tiempo operatorio (DMP: -0.46; IC 95\%: -0.77 a -0.14) con el uso de la embolización. Es importante mencionar que, en este último estudio, la media estandarizada de sangrados en los pacientes embolizados fue de $380 \mathrm{ml}(55-1,123)$, y para el tiempo operatorio fue de $200 \mathrm{~min}$ (150-360), lo cual fue muy parecido a nuestros resultados ${ }^{17}$. Por el contrario, en un metaanálisis previo de 470 pacientes, en el cual además se consideró la heterogeneidad de los estudios para el análisis de la DMP de las mismas variables, no se encontró diferencia significativa en el sangrado transoperatorio (DMP: -176.7 ; IC 95\%: -360.61 a 849), ni en el tiempo quirúrgico (DMP: -0.85 ; IC 95\%: -253 a $0.83)$, por lo que concluyó que no existe ninguna ventaja con la embolización ${ }^{18}$. 


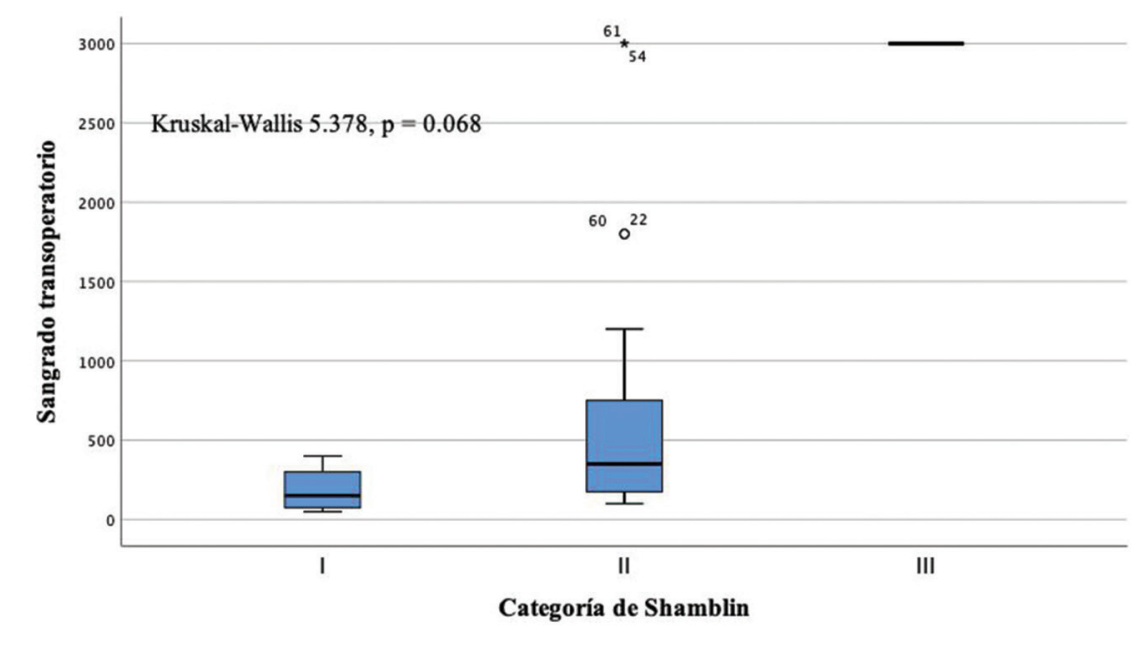

Figura 1. Comparación en el sangrado operatorio de acuerdo con la categoría de Shamblin.

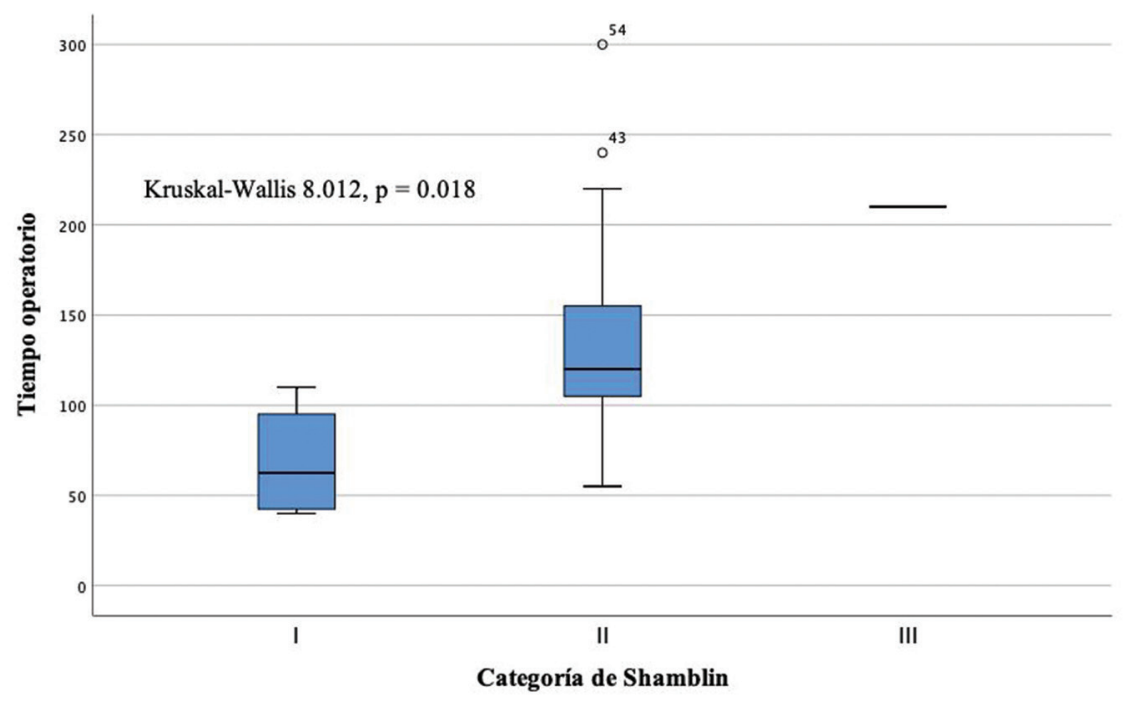

Figura 2. Comparación en el tiempo operatorio de acuerdo con la categoría de Shamblin.

Lo que resulta evidente es que existe una asociación significativa entre las complicaciones quirúrgicas y el grado de Shamblin. En el metaanálisis ya referido de Robertson, et al., en el que se analizaron los resultados del tratamiento de 4,743 TCC, el $97 \%$ de estos fueron tratados con cirugía y se encontró que la tasa de complicaciones, como el EVC, fueron significativamente mayores en los TCC Shamblin III (4\%), en comparación con los Shamblin I y II (1.89 y $2.71 \%$ respectivamente), así como para la lesión de nervios craneales (17\% para Shamblin III vs. 3.76 y $14.4 \%$ para Shamblin I y II respectivamente $)^{4}$. No obstante, otros estudios no han encontrado asociación en la clasificación de Shamblin y la tasa de complicaciones, pero sí en las dificultades técnicas durante la resección ${ }^{19}$. La tasa total de complicaciones en nuestro estudio, incluyendo la más grave (que fue el EVC en un paciente), representó el 5.5\%, lo cual fue muy similar a lo descrito en la literatura 20,21 . Se han descrito algunas otras estrategias para determinar con mayor exactitud el riesgo de complicaciones transoperatorias. En un estudio en el que se buscaron nuevos predictores de complicaciones transoperatorias, 


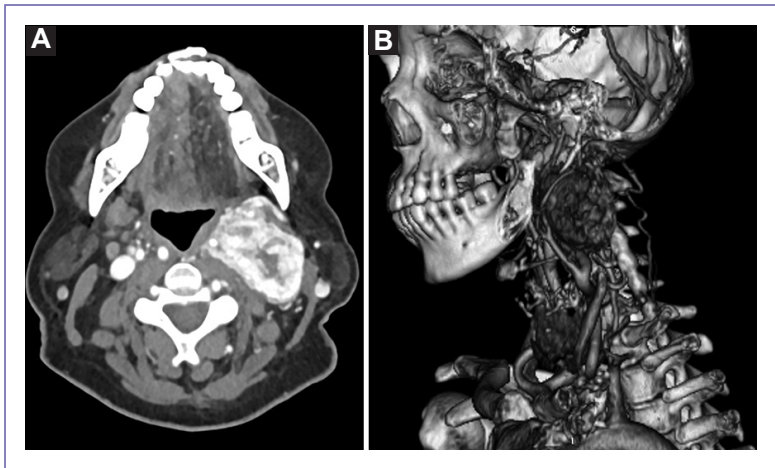

Figura 3. Angiotomografía de paciente con tumor del cuerpo carotídeo, categorizado como Shamblin III, tratado con radioterapia. A: corte axial. B: reconstrucción 3D.

se encontró que la media en la distancia del tumor a la base del cráneo medida en centímetros tuvo correlación inversamente proporcional con la pérdida sanguínea estimada y se asoció significativamente a la lesión de nervios craneales. Por su parte, el volumen tumoral calculado en $\mathrm{cm}^{3}$ tuvo una correlación directamente proporcional a la pérdida sanguínea estimada ${ }^{22}$.

También se han descrito otras técnicas quirúrgicas complementarias con el objetivo de disminuir el riesgo de complicaciones y sangrado transoperatorio; entre estas están: la disección craniocaudal, el uso de disector ultrasónico y energía bipolar avanzada, así como la colocación de stens preoperatorios y cortocircuitos transoperatorios. Todos ellos con un limitado número de casos, pero con resultados aparentemente buenos ${ }^{23-28}$.

En relación con la utilidad de la radioterapia en el tratamiento de los TCC, en una revisión sistemática que comparó los resultados de la cirugía contra 127 pacientes tratados con esta estrategia, se encontró que la tasa de control de la enfermedad, definida como regresión del tumor o ausencia de crecimiento, fue hasta del $94.5 \%$, la reducción del tumor se documentó en el $25 \%$, mientras que la ausencia de crecimiento representó el $69.3 \%$. Solo el $5.5 \%$ de los pacientes presentaron crecimiento del TCC. Las complicaciones del tratamiento con radioterapia estuvieron prácticamente ausentes, solo un paciente presentó síndrome de sistema nervioso central. Al comparar los resultados de la radioterapia con la cirugía, si bien no se encontró diferencia en el control de la enfermedad y las muertes, sí hubo diferencia en las complicaciones mayores y las lesiones de los nervios craneales $(p=0.047$ y 0.001 , respectivamente). Por esta razón, el estudio concluye que los pacientes con TCC voluminosos, que encasillan completamente la carótida o afectan el piso de cráneo deben ser tratados con radioterapia ${ }^{29}$. La cirugía radiocirugía robótica con CyberKnife ${ }^{\circledR}$ también ha sido descrita para el tratamiento de estos tumores, con resultados similares ${ }^{30}$.

De acuerdo con lo anterior y considerando el beneficio marginal o nulo de la embolización preoperatoria, la morbilidad de la cirugía y los buenos resultados con radioterapia, nosotros recomendamos que los pacientes con tumores Shamblin III deben ser tratados preferentemente con radioterapia ${ }^{31}$.

Por su parte, también se ha descrito la vigilancia de los TCC en algunos pacientes muy seleccionados; por ejemplo, aquellos que presentan una edad avanzada al momento el diagnóstico, tumores voluminosos y que sean asintomáticos, ya que la probabilidad de estabilidad es hasta del $42 \%$, otro gran porcentaje tendrá un crecimiento tumoral muy lento. Es importante el seguimiento estrecho en estos casos, para determinar si alguno de estos tumores requerirá de tratamiento en el futuro ${ }^{32}$.

Aquellos pacientes que no se sometieron a cirugía, ya sea por considerarse irresecables, o bien porque no aceptaron el procedimiento, fueron discutidos previamente de forma multidisciplinaria para discernir entre el tratamiento con radioterapia o mantenerlos en vigilancia. Después de decidir el tipo de conducta, cada paciente recibió una estrecha vigilancia con estudios de imagen con una frecuencia de al menos cada seis meses. Al momento del cierre de este estudio, no se presentaron casos con crecimiento del TCC con esta conducta.

En nuestro estudio, la frecuencia de tumores bilaterales fue mayor al $7 \%$ reportado en otras series; si bien la mayoría de ellos fueron sometidos a cirugía, la radioterapia y la vigilancia también fueron estrategias en el manejo de estos pacientes, logrando tasas de complicaciones bajas, similares al $37.5 \%$ reportado previamente $^{33}$.

En esta serie de casos no se presentó ningún TCC metastásico. Nosotros hemos evitado el uso del término «TCC maligno», ya que en la actualización de la clasificación de la Organización Mundial de Salud en el 2007 el mismo fue abandonado, ya que no existe un sistema histológico aceptado para la evaluación del comportamiento biológico agresivo de estos tumores ${ }^{34}$. Por lo tanto, en su lugar, el término «maligno» ha sido reemplazado por «metastásico» para referirse a tumores de comportamiento agresivo, los cuales se han reportado hasta en un $10 \%$ de los casos $^{35}$. 
Finalmente, si bien los resultados de las estrategias de tratamiento utilizada en nuestra serie parecen ser similares a lo reportado en la literatura, reconocemos que las limitaciones principales de los mismos son: que se trató de una serie de casos analizada de forma retrospectiva y que se presentaron pocos casos de TCC Shamblin I; esto último probablemente sea el resultado de un retraso en el diagnóstico o una referencia más tardía.

\section{Conclusiones}

Los TCC son neoplasias poco frecuentes, en su mayoría de comportamiento benigno y de lento crecimiento. La clasificación de Shamblin sigue siendo útil para predecir la resecabilidad y las complicaciones posoperatorias. Los TCC Shamblin I y II es la presentación más frecuente de estos tumores y en estos casos la cirugía es el tratamiento más adecuado, con una morbilidad aceptable. Según nuestros resultados, no se requiere embolización preoperatoria para llevar a cabo la resección del TCC. Por el contrario, en los tumores Shamblin III la radioterapia parece ser el tratamiento más adecuado, debido a sus buenos resultados y a que la cirugía se asocia a una alta tasa de complicaciones. Finalmente, la vigilancia activa es otra alternativa para aquellos pacientes que no aceptan el riesgo de la cirugía o en quienes no es posible dar radioterapia.

\section{Agradecimientos}

A los pacientes que confiaron en nosotros.

\section{Financiamiento}

Los autores declaran que no se recibió financiamiento para el desarrollo y la redacción de este escrito.

\section{Conflicto de intereses}

Los autores declaran que no existió conflicto de intereses durante el desarrollo y la redacción de este escrito.

\section{Responsabilidades éticas}

Protección de personas y animales. Los autores declaran que para esta investigación no se han realizado experimentos en seres humanos ni en animales.
Confidencialidad de los datos. Los autores declaran que han seguido los protocolos de su centro de trabajo sobre la publicación de datos de pacientes.

Derecho a la privacidad y consentimiento informado. Los autores han obtenido el consentimiento informado de los pacientes y/o sujetos referidos en el artículo. Este documento obra en poder del autor de correspondencia.

\section{Bibliografía}

1. Naik SM, Shenoy AM, Nanjundappa, et al. Paragangliomas of the carotid body: Current management protocols and review of literature. Indian $\mathrm{J}$ Surg Oncol. 2013;4(3):305-12.

2. Kaygusuz I, Karlidag T, Keles E, Yalcin S, Yüksel K. Carotid body tumor: Clinical features. J Craniofac Surg. 2015;26(7):e586-e589.

3. Shamblin WR, ReMine WH, Sheps SG, Harrison EG. Carotid body tumor (chemodectoma). Am J Surg. 1971;122(6):732-9.

4. Robertson V, Poli F, Hobson B, Saratzis A, Ross Naylor A. A systematic review and meta-analysis of the presentation and surgical management of patients with carotid body tumours. Eur J Vasc Endovasc Surg. 2019;57(4):477-86.

5. Hansen HS, Thomsen KA. Radiotherapy in glomus tumours (paragangliomas). A 25 year-review. Acta Otolaryngol Suppl. 1988;105(sup449):151-4.

6. Rodríguez-Cuevas S, López-Garza J, Labastida-Almendaro S. Carotid body tumors in inhabitants of altitudes higher than 2000 meters above sea level. Head Neck. 1998;20(5):374-78.

7. Inan HC, Yener HM, Karaman E, Kizilkiliç O, Cansiz H, Eker Ç. Role of preoperative embolization in surgical treatment of the carotid body paragangliomas. J Craniofac Surg. 2019;30(3):e267-e270.

9. Sevil FC, Tort M, Kaygin MA. Carotid body tumor resection: Long-term outcome of 67 cases without preoperative embolization. Ann Vasc Surg. 2020;67:200-7.

10. Mascia D, Esposito G, Ferrante A, Grandi A, Melissano G, Chiesa R. Carotid body tumor contemporary management in a high-volume center. J Cardiovasc Surg (Torino). 2020;61(4):459-66.

11. Kuchakulla M, Shah AH, Armstrong V, Jernigan S, Bhatia S, Niazi TN. Multimodal management of pediatric carotid body tumors: a systematic review and case illustrations. J Neurosurg Pediatr. 2018;23(3):325-32.

12. Han T, Wang S, Wei X, Xie Y, Sun Y, Sun H, et al. Outcome of surgical treatment for carotid body tumors in different shambling type without preoperative embolization: A single-center retrospective study. Ann Vasc Surg. 2020;63:325-31.

13. Liu J, Li Y, Yang L, Cai H. Surgical resection of carotid body tumors with versus without preoperative embolization: Retrospective case-control study. Head Neck. 2018;40(12):2590-5.

14. Zhang T, Jiang W, Li Y, Li B, Yamakawa T. Perioperative approach in the surgical management of carotid body tumors. Ann Vasc Surg. 2012;26(6):775-82.

15. Power AH, Bower TC, Kasperbauer J, Link MJ, Oderich G, Cloft H, et al. Impact of preoperative embolization on outcomes of carotid body tumor resections. J Vasc Surg. 2012;56(4):979-89.

16. Texakalidis P, Charisis N, Giannopoulos S, Xenos D, Rangel-Castilla L, Tassiopoulos AK, et al. Role of preoperative embolization in carotid body tumor surgery: A systematic review and meta-analysis. World Neurosurg. 2019;129:503-513.e2.

17. Jackson RS, Myhill JA, Padhya TA, McCaffrey JC, McCaffrey TV, Mhaskar RS. The effects of preoperative embolization on carotid body paraganglioma surgery: A systematic review and meta-analysis. Otolaryngol Head Neck Surg. 2015;153(6):943-50.

18. Abu-Ghanem S, Yehuda M, Carmel NN, Abergel A, Fliss DM. Impact of preoperative embolization on the outcomes of carotid body tumor surgery: A meta-analysis and review of the literature: Preoperative embolization of carotid body tumor. Head Neck. 2016;38(S1):E2386-E2394.

19. Law Y, Chan Y, Cheng S. Surgical management of carotid body tumor - Is Shamblin classification sufficient to predict surgical outcome? Vascular. 2017;25(2):184-9.

20. Dorobisz K, Dorobisz T, Temporale H, Zato®ski T, Kubacka M, Chabowski M, et al. Diagnostic and therapeutic difficulties in carotid body paragangliomas, based on clinical experience and a review of the literature. Adv Clin Exp Med. 2016;25(6):1173-7.

21. Amato B, Serra R, Fappiano F, Rossi R, Danzi M, Milone M, et al. Surgical complications of carotid body tumors surgery: a review. Int Angiol. 2015;34(6 Suppl 1):15-22. 
22. Kim GY, Lawrence PF, Moridzadeh RS, Zimmerman K, Munoz A, Luna-Ortiz K, et al. New predictors of complications in carotid body tumo resection. J Vasc Surg. 2017;65(6):1673-9.

23. Paridaans MPM, van der Bogt KEA, Jansen JC, Nyns EC, Wolterbeek $R$ van Baalen JM, et al. Results from craniocaudal carotid body tumor resection: Should it be the standard surgical approach? Eur J Vasc Endovasc Surg. 2013;46(6):624-9.

24. Ferreira J, Canedo A, Braga S, Vasconcelos J, Gouveia R, Martins V, et al. Carotid body tumours resection with ultrasound dissector. Chin Med J (Engl). 2013;126(3):586-8.

25. Luna-Ortiz K, Villavicencio-Valencia V, Carmona-Luna T, Cano-Valdez AM Herrera Gómez A. Resección de tumor de cuerpo carotídeo con LigaSureß. Acta Otorrinolaringol Esp. 2010;61(1):6-11.

26. Piazza P, Di Lella F, Bacciu A, Di Trapani G, Ait Mimoune H, Sanna M. Preoperative protective stenting of the internal carotid artery in the management of complex head and neck paragangliomas: Long-term results. Audiol Neurotol. 2013;18(6):345-52.

27. Zeng $G$, Zhao J, Ma Y, Huang $B$. Resection of carotid body tumors and the additional choice of intraoperative shunt in complicated tumors. Ann Vasc Surg. 2012;26(4):511-5.

28. Scanlon JM, Lustgarten JJ, Karr SB, Cahan Jl. Successful devascularization of carotid body tumors by covered stent placement in the external carotid artery. J Vasc Surg. 2008;48(5):1322-4
29. Suárez C, Rodrigo JP, Mendenhall WM, Hamoir M, Silver CE, Grégoire V, et al. Carotid body paragangliomas: a systematic study on management with surgery and radiotherapy. Eur Arch Otorhinolaryngol. 2014; 271(1):23-34.

30. Bianchi LC, Marchetti M, Brait L, Bergantin A, Milanesi I, Broggi G, et al. Paragangliomas of head and neck: a treatment option with CyberKnife radiosurgery. Neurol Sci. 2009;30(6):479-85.

31. Gilbo P, Morris CG, Amdur RJ, Werning JW, Dziegielewski PT, Kirwan J, et al. Radiotherapy for benign head and neck paragangliomas: A 45-year experience: RT for Head and Neck Paragangliomas. Cancer. 2014;120(23):3738-43.

32. Langerman A, Athavale SM, Rangarajan SV, Sinard RJ, Netterville JL. Natural history of cervical paragangliomas: outcomes of observation of 43 patients. Arch Otolaryngol Head Neck Surg. 2012; 138(4):341-5.

33. Bobadilla-Rosado LO, Garcia-Alva R, Anaya-Ayala JE, Peralta-Vazquez C, Hernandez-Sotelo K, Luna $L$, et al. Surgical management of bilateral carotid body tumors. Ann Vasc Surg. 2019;57:187-93.

34. WHO Classification of Tumours of Endocrine Organs. 4th edition. International Agency for Research on Cancer; 2017.

35. Lam AK. Update on adrenal tumours in 2017 World Health Organization (WHO) of endocrine tumours. Endocr Pathol. 2017;28(3):213-27. 